\title{
Optimum Scheduling the Electric Distribution Substations with a Case Study: An Integer Gaining-Sharing Knowledge-Based Metaheuristic Algorithm
}

\author{
Said Ali Hassan, ${ }^{1}$ Khalid Alnowibet, ${ }^{2}$ Prachi Agrawal, ${ }^{3}$ and Ali Wagdy Mohamed $\mathbb{D}^{4,5}$ \\ ${ }^{1}$ Department of Operations Research and Decision Support, Faculty of Computers and Artificial Intelligence, Cairo University, \\ Giza, Egypt \\ ${ }^{2}$ College of Science, Department of Statistics and Operations Research, King Saud University, Riyadh, Saudi Arabia \\ ${ }^{3}$ Department of Mathematics and Scientific Computing, National Institute of Technology Hamirpur, Hamirpur 177005, \\ Himachal Pradesh, India \\ ${ }^{4}$ Operations Research Department, Faculty of Graduate Studies for Statistical Research, Cairo University, Giza 12613, Egypt \\ ${ }^{5}$ Wireless Intelligent Networks Center (WINC), School of Engineering and Applied Sciences, Nile University, Giza, Egypt
}

Correspondence should be addressed to Ali Wagdy Mohamed; aliwagdy@gmail.com

Received 15 October 2020; Revised 26 October 2020; Accepted 30 October 2020; Published 8 December 2020

Academic Editor: Ahmed Mostafa Khalil

Copyright (c) 2020 Said Ali Hassan et al. This is an open access article distributed under the Creative Commons Attribution License, which permits unrestricted use, distribution, and reproduction in any medium, provided the original work is properly cited.

\begin{abstract}
This work is dedicated to the economic scheduling of the required electric stations in the upcoming 10-year long-term plan. The calculation of the required electric stations is carried out by estimating the yearly consumption of electricity over a long-time plan and then determining the required number of stations. The aim is to minimize the total establishing and operating costs of the stations based on a mathematical programming model with nonlinear objective function and integer decision variables. The introduced model is applied for a real practical case study to conclude the number of yearly constructed stations over a long-term plan in the electricity sector in Jeddah City, Saudi Arabia. The current planning method is based only on intuition by constructing the same number of required stations in each year without searching for better solutions. To solve the introduced mathematical model, a novel recent gaining sharing knowledge-based algorithm, named GSK, has been used. The Augmented Lagrangian Method (ALM) is applied to transform the constrained formulation to become unconstrained with penalization to the objective function. According to the obtained results of the real case study, the proposed GSK with ALM approved an ability to solve this case with respect to convergence, efficiency, quality, and robustness.
\end{abstract}

\section{Introduction}

In the coming years, the population projection is expected to rise worldwide, and this should be accomplished through an enough increase in the supply of electricity to cover the anticipated higher demand.

Expert opinions suggest that the expected load demand is a key factor needed for preparing the potential power needs. Planning authorities around the world state that the forecast of the population is to be continuously increasing [1]. This increase should be balanced by an enough increase in the supply of electricity.
Since electricity demand is in a direct relation to the anticipated population growth, the electricity company should support the current network by installing additional stations to meet the growing demand [2]. The infrastructure utilities demand such as water, electricity, wastewater, and communication would surpass proportion to the population growth and the expansion of new urban areas. The responsible authorities are required to support the corresponding networks by installing new stations to meet the growing demand and prevent business disruption and economic losses due to electricity failures [3]. 
The current solution procedure is based mainly on the real immediate needs constrained by the available resources. This can lead, either, to the construction of few stations while the resources allow for excess, or, to the construction of less than required due to budget constraints. For these purposes, a comprehensive analytical method is badly needed by the electricity company to determine the ideal decisions concerning the yearly number of installed stations over the longtime plan.

The current protocol is fully dependent on experience and intuition. This decision-making process is completely subjective and, in most cases, will lead to excess costs of the order of million dollars for building and operating the required stations. The decision-making bodies need a detailed analytical method to determine the best decisions about establishing the electric stations along the scheduling period. This study is considered as a basis for planning and scheduling these utilities and reaches the optimum solutions supported by mathematical bases.

On the contrary, over the last three decades, metaheuristic algorithms become popular for solving the high dimensional, complex optimization problems and have shown ability to find near optimal solutions. Recently, Mohamed et al. [4] introduced a new nature-inspired procedure, gaining and sharing knowledge GSK. The GSK procedure is tested over thirty test problems from CEC2017 benchmark function in different dimensions. It is also applied to IEEE-CEC2011 problems to solve real-practical problems and is compared to more than ten state-of-the-art metaheuristic algorithms. Obtained outputs indicate significant overcoming of GSK over other algorithms with respect to robustness, convergence, and ability to find solutions to used problems. GSK consists of two phases:

(i) Junior GSK phase

(ii) Senior GSK phase

In the first phase, junior or beginners gain knowledge from their small networks such as from family members, relatives, and friends. They want to share their knowledge with other people who may not belong to their network due to curiosity of exploring others. Also, they could not differentiate between good or bad people. Similarly, in the senior gaining and sharing phase, the people have the experience to judge others, and they can categorize them. Senior people gain their knowledge from their colleagues, social friends, or many others and share their views or opinions according to their experience and can enhance their learning [4].

Because the introduced formulation is so complex as it is nonlinear integer programming one, an enhanced constrained GSK algorithm is suggested to handle the given NL integer GP formulation.

1.1. Contribution. The current planning method for the establishing of the electric transmission substations in Saudi Electricity Company is based on intuition and experience by constructing the same number of demanded substations in each year without searching for better solutions. This procedure does not lead to the optimum solution and in most cases, causes excess of expenses all over the longplanning horizon. This paper aims at achieving an economic scheduling of the required electric distribution substations in a long term. The objective is to minimize the total establishing and operating costs of the stations on the planning horizon based on a nonlinear integer mathematical programming model.

The introduced model is applied for a real practical case study to conclude the number of yearly constructed stations over a long-term plan in the electricity sector in Jeddah city, Saudi Arabia.

To solve the introduced mathematical model, a novel recent gaining-sharing knowledge-based algorithm, named GSK, has been used.

The Augmented Lagrangian Method (ALM) is applied to transform the constrained formulation to become unconstrained with penalization to the objective function. According to the obtained results of the real case study, the proposed GSK with ALM approved an ability to solve this case with respect to convergence, efficiency, quality, and robustness. The total cost savings by using such a scientific approach rather than the traditional used method in practice is more than $\$ 20$ million.

This scientific approach for modelling and solving such practical problems can be easily applied by managers in practice for this and for similar scheduling problems. In this manner, they will always reach the optimum solution and save time and excess costs.

The rest of this work is organized in the following manner. Section 2 is devoted for the literature review of the current problem. Section 3 introduces economic scheduling of the electric distribution substation mathematical model. Section 4 introduces a practical application case study for the problem in Jeddah, Saudi Arabia. A proposed IGSK approach and computational results of the problem are presented in Sections 5 and 6, respectively. The conclusions and suggested research points are summed up in Section 7.

\section{Literature Review}

Several studies such as Al-Saleh and Taleb [5] reveal a dramatical jump in electricity consumption throughout the world. Ignoring effective designs of buildings and the lack of "time-of-use" of electric power contribute about 80 percent of used electricity for air conditioning and cooling. And then, as demand is at its highest, electricity shortages become acute during the summer season [6].

Gelling et al. [7] categorized the forecasting of electrical demand into three types: short, medium, and long term. Short-term forecast is performed upon few hours, medium type is for few weeks up to few years, and long term is influenced mainly by economic factors and is used for 5 to 15 and up to 25 years.

Parols et al. [8] stated that forecasting the demanded load in a service area is a basic factor of electric resource planning. Using a minimum cost schedule, resources can be organized to cover the expected demand based on the outcome of such projections. Asset preparation is usually carried out subject 
to various uncertainties where the forecast of load demand represents a major part of uncertainties.

$\mathrm{Lu}$ and Hsu [9] work is to study the objective to minimize the voltage deviations in the reactive power flow from the desired values. A dynamic programming-based approach is used.

Yixin et al. [10] studied the distribution network planning, and they stated that the loads received in different years within each sector are different, so efficient planning is a dynamic programming formulation.

Nagasaka and Al-Mamun [11] predicted peak loads using the function networks of the radial basis. The prediction of nine firms in Japan reflects current and future trends. The research concentrates on economic data influencing long-forecasting demand for electrical loads. The used data are real annual load and incremental increasing percent in past years.

Al Ghamdi [12] concentrated on the electricity sector in Jeddah for a timetable of 10 years-time horizon. He extends the analysis to include some parametric findings investigating the effect on the optimal solution obtained by adjusting the problem parameters.

Hassan et al. [13] show that demand of electric power in Saudi Arabia has risen dramatically related to the previous decades. This is a result of rapidly growing population, economic growth, and shortage of energy protection activities. They use ANN to predict the required electrical distribution stations in Jeddah. A dynamic programming formulation is then developed for scheduling number of yearly installed stations for the prescribed time period to minimize the overall cost of establishing and operating the determined stations.

El Quliti et al. [14] based this study in the city of Jeddah. The mentioned work is for scheduling the necessary number of substations in a long-term period using a dynamic programming model. The calculation is based on determining the number of stations to be built to minimize the total costs over the planning horizon.

The work of El-Quliti and Kabli [15] is dedicated to planning the optimum number of electrical substations required for a long-term period. The model also provides a parametric sensitivity analysis to detect the possible variations in basic parameters on the obtained results.

The dynamic programming approach is generally difficult to understand, and it does not have a general feature for all applications, instead each application has its structure. The dynamic programming divides problems into several decision phases; the result of a decision at one stage affects the decisions at each of the next stages. There is an abundance of calculations where any minor error in any would result in an incorrect result.

In fact, there is no general algorithm (such as the simplex method for linear programming) that can be programmed to solve all sorts of applications. Additionally, there are no code packages for solving various dynamic programming problems, such as the case for mathematical programming models.

To overcome these difficulties occurring in solving optimization problems, metaheuristic procedures are originated in the latest three decades. Algorithms based on metaheuristic approaches are used to handle many real-life applications because they are simple, able to detect near optimal solutions, and easy to implement. It is divided into the following four groups:

(i) Evolutionary algorithms (based on natural evolution and inspired from biology): many metaheuristic algorithms come under this category such as evolutionary programming, genetic algorithm [16], differential evolution [17], and tabu search [18].

(ii) Swarm-based algorithms (based on the behaviour of social animals (school of fishes, birds etc.)): swarmbased procedures are devoted for solving more complicated problems. Optimization using particle Swarm [19], ant colony [20], and Whale algorithm [21] are in this group.

(iii) Physics-based algorithms: it is outstanding with regulations of governing a natural phenomenon. Simulated annealing [22], harmony search [23], water cycle algorithm [24], etc. are the examples of physics-based techniques.

(iv) Human-based algorithms: these algorithms depend on the human behaviour or activity. Based on the physical activities or nonphysical of human, these kinds of techniques are developed. There are very few algorithms which are in human based category, e.g., human-inspired algorithm [25] and teachinglearning based optimization [26].

Metaheuristic algorithms have been applied to various fields and solved the real-time problems. A comparative study has been carried out among evolutionary algorithms, tabu search, and simulated annealing [27], and evolutionary strategy and genetic algorithm are compared to solve the mechanical design problem with different types of constraints [28]. El-Qulity and Mohamed [29] solved a higher education admission problem using a nonlinear goal programming model with integer decision variables using a modified differential evolution algorithm. The obtained results show the robustness and efficiency of the modified differential evolution. Besides, they applied advanced versions of DE-based algorithms to solve many other real-world applications [30-41]. Gomes [42] solved the truss mass optimization problem using particle swarm procedure with nonlinear dynamic constraints. Wang and Watada [43] solved a real capacitated location situation including fuzzy parameters using hybrid algorithms. Metaheuristic algorithm provides easy and simple methods to solve the highdimensional and nonlinear complex optimization problem. Due to simplicity of algorithms, Haddad et al. [18] utilized a procedure for water cycle to find the optimal strategies for the Karon-4 reservoir system. For comparison evaluation, the procedure is evaluated with genetic algorithm and the obtained solution deduces that it is more efficient and reliable algorithm. None of the abovementioned algorithm is used to solve the economic scheduling of electric transmission station problems. 
Therefore, based on the characteristics of GSK, integer GSK (IGSK) based on algorithms for finding optimum solutions is introduced to handle the problem with nonlinear programming formulation having integer decision variables. For the integer decision variables, IGSK comprises integer mutation which converts a whole number to its nearest integer. It is applied to junior and senior gainingsharing stage. The proposed IGSK is a simple modification in the developed GSK to handle the problem with nonlinear formulation for economic scheduling of the electric transmission substations.

\section{Formulation of the Mathematical Model for the Problem}

\subsection{Known Parameters}

$B_{y}^{n}=$ building cost of one substation in year $y$ when buying $n$ substations in that year, and this value does not mean the product $B_{y}^{n}$ is generally a nonlinear function producing the unit building cost as a function of the number of established substations $x_{y}$ in year $y$, $y=1,2, \ldots, N$

$N=$ number of planning years

$O_{y}=$ annual Operations and Maintenance (O\&M) costs for one substation in year $y$

$d_{y}=$ yearly demanded substations in year $y$, and this value depends mainly on the increased electricity consumption each year due to new buildings and projects consuming electric power

$M_{y}=$ maximum number of substations that can be built in year $y$ due to the resource's constraints.

3.2. Decision Variables. The decision variables to be determined in this problem are the number of substations to be built each year in the long-term planning horizon, denoted by $x_{y}, y=1,2, \ldots, N$.

\subsection{Problem Constraints}

3.3.1. Resource Constraints. The maximum number of substations to be built each year $y$ of the planning horizon should not exceed the available resources. Resources comprise the budget, specialized employees, equipment, and consumed materials:

$$
0 \leq x_{y} \leq M_{y}, \quad y=1,2, \ldots \ldots, N
$$

where $M_{y}=$ maximum number of substations to be built in year $y, y=1,2, \ldots, N$.

3.3.2. Demand Constraints. The accumulated number of established substations till year $y$ should be greater or equal to the accumulated request in all previous years preceding that year $y$ :

$$
\sum_{c=1}^{y} x_{c} \geq \sum_{c=1}^{y} d_{c}, \quad y=1,2, \ldots N
$$

3.3.3. Nonnegativity and Integer Constraints. All $x_{y}$ are nonnegative integers:

$$
y=1,2, \ldots N
$$

3.3.4. The Objective Function. The ultimate objective for the problem is to determine the decision variables $x_{1}, x_{2}, x_{3}, \ldots, x_{N}$ such that the total costs of building and operating the electric transmission/distribution substations over the long planning horizon are minimized, so we have

$$
\operatorname{Min} z=\sum_{y=1}^{N}\left[B_{y}(x) \cdot x_{y}+\left(x_{y} \cdot \sum_{j=y+1}^{N} O_{j}\right)\right] \text {. }
$$

Defining $B_{\mathrm{y}}(x)$ as a function of $x_{y}$, then the objective will have the form

$$
\operatorname{Min} z=\sum_{y=1}^{N}\left[f_{y}\left(x_{y}\right) \cdot x_{y}+\left(x_{y} \cdot \sum_{j=y+1}^{N} O_{j}\right)\right],
$$

where $f_{y}\left(x_{y}\right)$ is a function of $x_{y}, y=1,2, \ldots, N$. The relation of $\left[f_{y}\left(x_{y}\right)\right]$ and $x_{y}$ is a table of the values representing the number of substations and the cost of each one in each year of the planning horizon. Taking into considerations that the cost of one substation will increase year by year, while there will be some discounts for buying a greater number of substations, see Table 1, which represents these data.

It is required to represent the set of data points in Table 1 for each year $y, y=1,2, \ldots, N:\left(1, B_{y}^{1}\right),\left(1, B_{y}^{2}\right), \ldots,\left(1, B_{y}^{n}\right)$ with a polynomial of degree $(n-1)$, and this can be done using an appropriate polynomial interpolation computer program [44]. Such a polynomial exists and is unique [45].

\section{A Real Application Case Study}

Jeddah is the second big city in the Kingdom of Saudi Arabia, and it has a very important location near to the two holy mosques, with lot of commercial, industrial, and architectural development (Figure 1). As per long-term strategy plan for Jeddah, the population forecast is expected to be continuously increasing within the next coming years.

The Saudi Electricity Company (SEC) in Jeddah is facing a big challenge to balance the upcoming needed demands and the budget availability. The major concern of SEC is to provide adequate supply of electricity to the public and industry in each area in Jeddah city. Because the power demand is going to exceed the electricity endurance in a direct proportion to the definite growth in population and to the building environment expand, extensively SEC will be required to reinforce the network by the installation of new substations in these areas.

The needed number of electric transmission/distribution substations for a long-term time horizon of the upcoming 
TABLE 1: The number of substations and the unit building cost.

\begin{tabular}{lccccc}
\hline \multirow{2}{*}{ Number of substations } & \multicolumn{4}{c}{ Cost of building one substation } \\
\\
\hline $\mathbf{1}$ & Year 1 & Year 2 & Year $y$ & $\ldots$ & Year $N$ \\
$\mathbf{2}$ & $B_{1}^{1}$ & $B_{2}^{1}$ & $B_{y}^{1}$ & $\ldots$ & $B_{N}^{1}$ \\
$\mathbf{3}$ & $B_{1}^{2}$ & $B_{2}^{2}$ & $B_{y}^{2}$ & $\ldots$ & $B_{N}^{2}$ \\
$\ldots$ & $B_{1}^{3}$ & $B_{2}^{3}$ & $B_{y}^{3}$ & $\ldots$ & $B_{N}^{3}$ \\
$\ldots$ & $\ldots$ & $\ldots$ & $\ldots$ & $\ldots$ & $\ldots$ \\
$N$ & $\ldots$ & $\ldots$ & $\ldots$ & $\ldots$ \\
\hline
\end{tabular}

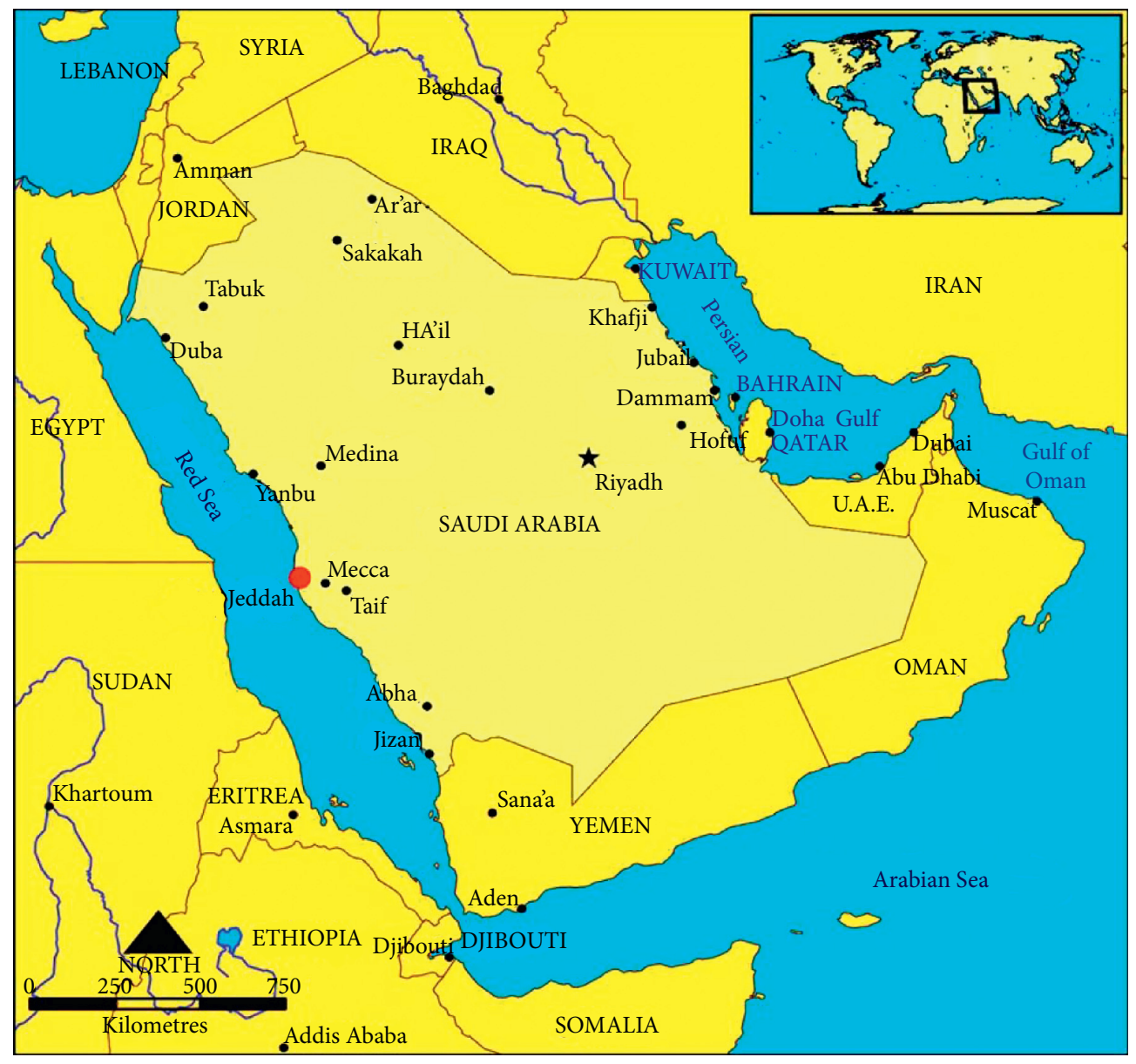

Figure 1: Location of Jeddah city in Saudi Arabia.

10 years is predicted based on the electricity total consumption in each year and then defining the number of substations needed for that year.

There are many parameters affecting the formulation of the problem, these parameters are

(i) The initial value of the substation building cost

(ii) The percentage of yearly increase in the substation building cost

(iii) The percentage of discount for buying more than one substation

(iv) Initial value of the operation cost (v) The percentage of yearly increase in the operation cost

It is required to provide SEC with a systematic procedure to arrive at the optimum decision for the number of substations to be built each year during the planning time horizon of the coming 10 years.

The yearly demanded substations in the following 10 years $d_{y}=3,1,3,3,2,2,2,3,3,3$. The building cost for one substation in the first year $B_{1}^{1}$ is estimated to be 50 million Saudi Riyals (SR) and is assumed to increase by $7 \%$ annually over the next 10 years; there is a discount of $5 \%$ for 2 building substations and $10 \%$ for 3 substations in all years of the 
planning period. Operation and maintenance cost $\left(O_{y}\right)$ starts from 3.00 million SR in the first year and increases by $7 \%$ annually for the next 10 years.

Table 2 represents the building costs for one substation over the next 10 years in case of purchasing 1,2 , and 3 substations which is the maximum number of possible established substations due to the resource's constraints. The table also represents the yearly demand $\left(d_{y}\right)$, the accumulated yearly demand $\left(D_{y}\right)$, the operating and maintenance $(\mathrm{O} \& \mathrm{M})$ costs $\left(O_{y}\right)$, and the cumulative O\&M costs $\left(\sum O_{y}\right)$ for the 10 upcoming years, where

$$
\begin{aligned}
& D_{y}=\left(\sum_{c=1}^{y} d_{c}\right), \quad y=1,2, \ldots, N, \\
& \sum O_{y}=\left(\sum_{j=y+1}^{10} O_{j}\right), \quad y=1,2, \ldots N .
\end{aligned}
$$

The following mathematical model is obtained according to the given data of the case study.

4.1. Resource Constraints. The available resources allow only for a maximum of $M_{y}=3$ substations to be built in any year of the planning horizon. Applying formula (1), then we have

$$
0 \leq x_{y} \leq 3, \quad y=1,2, \ldots \ldots, 10 .
$$

4.2. Demand Constraints. The accumulated number of established substations till year $y$ should be greater or equal to the accumulated demand in all previous years preceding that year $y$. Applying formula (2), then we have

In year $1, x_{1} \geq 3$ (demanded substations in the first year)

In year $2, x_{1}+x_{2} \geq 4$ (accumulated number of demanded substations in the first 2 years)

In year $3, x_{1}+x_{2}+x_{3} \geq 7$ (accumulated number of demanded substations in the first 3 years) and so on for the next years

4.3. Integer Constraints. Applying formula (3), then we have $x_{1}, x_{1}, x_{2}, x_{3}, \ldots, x_{10}$ as integers $\geq 0$.

4.4. The Objective Function. A suitable software package, MyCurveFit [46], is used to interpolate a quadratic polynomial in formula (5) for the discounted prices of substations in each year. Figure 2 presents the data entry screen and the output of the interpolation for the first year where the data points are $(1,50.0),(2,47.5)$, and $(3,45.0)$, as shown in the first-year data in Table 2. The obtained polynomial equation is $\left(52.5-2.5 x_{1}\right)$ and other polynomial equations are obtained for the other years using the mentioned computer package.

The curve fitting for all the 10 years are obtained and substituted to form the objective function as follows:

$$
\begin{aligned}
\operatorname{Min} z= & \sum_{y=1}^{10}\left[\left(f_{y}\left(x_{y}\right) \cdot x_{y}+\left(x_{y} \cdot \sum_{j=y+1}^{10} O_{j}\right)\right)\right] \\
= & {\left[\left(52.5 x_{1}-2.5 x_{1}^{2}\right)+\left(38.38 x_{1}\right)\right] } \\
& +\left[\left(56.16 x_{2}-2.655 x_{2}^{2}-0.005 x_{2}^{3}\right)+\left(35.17 x_{2}\right)\right]+ \\
& +\left[\left(60.11 x_{3}-2.86 x_{3}^{2}\right)+\left(31.74 x_{3}\right)\right] \\
& +\left[\left(64.31 x_{4}-3.06 x_{4}^{2}\right)+\left(28.06 x_{4}\right)\right]+ \\
& +\left[\left(68.80 x_{5}-3.255 x_{5}^{2}-0.005 x_{5}^{3}\right)+\left(24.13 x_{5}\right)\right]+ \\
& +\left[\left(73.62 x_{6}-3.485 x_{6}^{2}-0.005 x_{6}^{3}\right)+\left(19.92 x_{6}\right)\right]+ \\
& +\left[\left(78.79 x_{7}-3.75 x_{7}^{2}\right)+\left(15.42 x_{7}\right)\right] \\
& +\left[\left(84.29 x_{8}-3.99 x_{8}^{2}-0.005 x_{8}^{3}\right)+\left(10.67 x_{8}\right)\right]+ \\
& +\left[\left(90.19 x_{9}-4.275 x_{9}^{2}-0.005 x_{9}^{3}\right)+\left(5.52 x_{9}\right)\right] \\
& +\left[\left(96.5 x_{10}-4.575 x_{10}^{2}-0.005 x_{10}^{3}\right)\right] \\
= & \left(90.88 x_{1}-2.5 x_{1}^{2}\right)+\left(91.33 x_{2}-2.655 x_{2}^{2}-0.005 x_{2}^{3}\right) \\
& +\left(91.85 x_{3}-2.86 x_{3}^{2}\right)+ \\
& +\left(92.37 x_{4}-3.06 x_{4}^{2}\right)+\left(92.93 x_{5}-3.255 x_{5}^{2}-0.005 x_{5}^{3}\right)+ \\
& +\left(93.54 x_{6}-3.485 x_{6}^{2}-0.005 x_{6}^{3}\right) \\
& +\left(94.21 x_{7}-3.75 x_{7}^{2}\right)+ \\
& +\left(94.96 x_{8}-3.99 x_{8}^{2}-0.005 x_{8}^{3}\right) \\
& +\left(95.71 x_{9}-4.275 x_{9}^{2}-0.005 x_{9}^{3}\right) \\
& +\left(96.5 x_{10}-4.575 x_{10}^{2}-0.005 x_{10}^{3}\right) .
\end{aligned}
$$

\section{Methodology}

This part represents the methodology used in solving the prescribed problem. Section 5.1 presents the detailed description of proposed algorithm IGSK and Section 5.2 describes the constraint handling approach.

5.1. Integer Gaining-Sharing Knowledge-Based Optimization Algorithm (IGSK). IGSK is suggested to handle the integer decision variables. The GSK algorithm is modified with an integer mutation leading to the development of a new Integer Gaining-Sharing Knowledge- (IGSK-) based optimization algorithm. Integer variables are handled with round operator, i.e., round $(a)$, which rounds the number $a$ to the nearest integer. The mathematical formulation of IGSK is described as follows.

Step 1: initially, persons' number is assumed as NOP (number of population). Let $x_{i}(i=1,2, \ldots, \mathrm{NOP})$ be an individual which is defined as $x_{i j}=\left(x_{i 1}, x_{i 2}, \ldots, x_{\mathrm{id}}\right)$, where $d$ is the count of discipline fields and $f_{i}(i=1,2, \ldots, \mathrm{NOP})$ is the corresponding objective function values. The initial population is generated as 
TABLE 2: Building and O\&M costs for one substation in different years.

\begin{tabular}{lcccccccccc}
\hline $\mathbf{S} / \mathbf{S}$ & $\mathbf{1}$ & $\mathbf{2}$ & $\mathbf{3}$ & $\mathbf{4}$ & $\mathbf{5}$ & $\mathbf{6}$ & $\mathbf{7}$ & $\mathbf{8}$ & $\mathbf{9}$ & $\mathbf{1 0}$ \\
\hline $\mathbf{1}$ & 50.0 & 53.50 & 57.25 & 61.25 & 65.54 & 70.13 & 75.04 & 80.29 & 85.91 & 91.92 \\
$\mathbf{2}$ & 47.5 & 50.83 & 54.39 & 58.19 & 62.27 & 66.62 & 71.29 & 76.28 & 81.62 & 87.33 \\
$\mathbf{3}$ & 45.0 & 48.15 & 51.52 & 55.13 & 58.99 & 63.11 & 67.53 & 72.26 & 77.32 & 82.73 \\
$\mathbf{d}_{y}$ & 3 & 1 & 3 & 3 & 2 & 2 & 2 & 3 & 3 & 3 \\
$\mathbf{D}_{y}$ & 3 & 4 & 7 & 10 & 12 & 14 & 16 & 19 & 22 & 25 \\
$\mathbf{O}_{y}$ & 3.0 & 3.21 & 3.43 & 3.68 & 3.93 & 4.21 & 4.50 & 4.82 & 5.15 & 5.52 \\
$\mathbf{\Sigma}_{y}$ & 38.38 & 35.17 & 31.74 & 28.06 & 24.13 & 19.92 & 15.42 & 10.67 & 5.52 & 0.00 \\
\hline
\end{tabular}

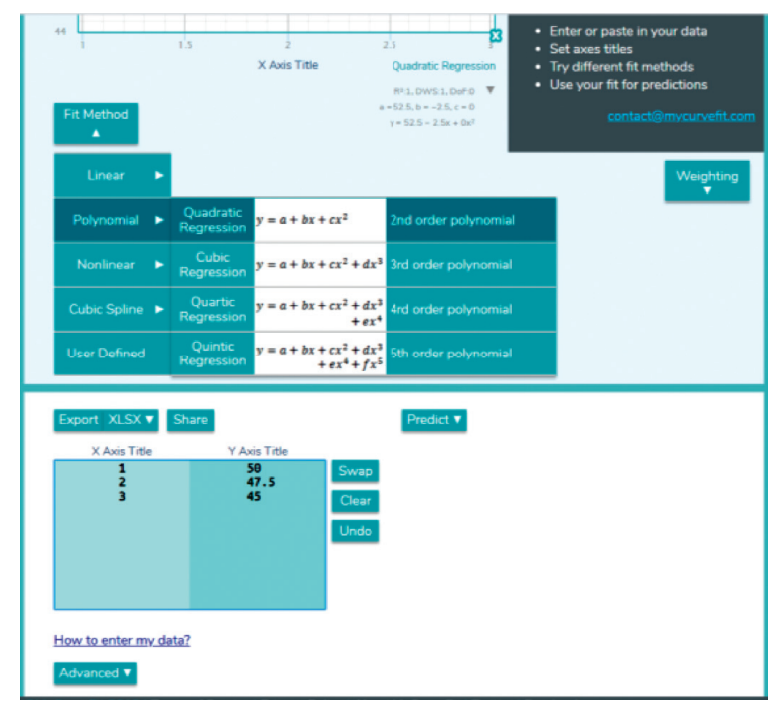

(a)

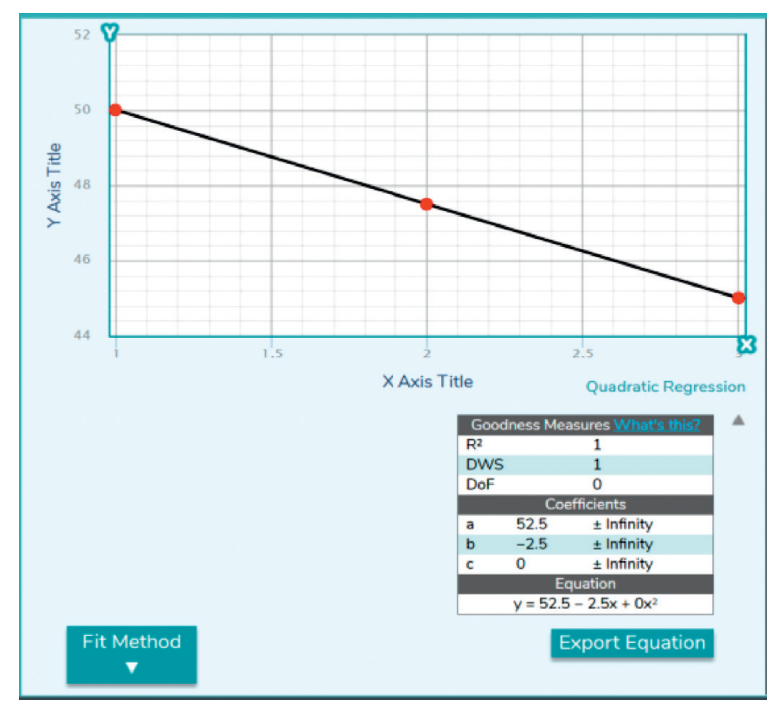

(b)

Figure 2: The data entry and the output screens of the MyCurveFit Software.

$$
x_{i j}=\operatorname{round}(l b+\operatorname{rand} *(u b-l b)) \text {, }
$$

where $u b$ and $l b$ and represent the topmost and least limits of the optimization task.

Step 2: at starting of the search for the solution, the dimensions for the junior or senior phase must be set on. The dimensions' number that should be changed or updated during both phases should be computed by increasing and decreasing formula:

$$
\begin{aligned}
& d_{\text {junior }}=d \times\left(1-\frac{G}{\mathrm{Gen}}\right)^{k}, \\
& d_{\text {senior }}=d-d_{\text {junior }},
\end{aligned}
$$

where $k$ is the knowledge factor and a positive real number which controls the knowledge rate, Gen is the topmost count of generations, and $G$ represents the number of generations.
Step 3 (junior gaining and sharing stage): it considers at beginners' gain knowledge from their friends, family, etc. and share their views with other people who may or may not belong to their networks. This phase considers further two subphases as follows:

(i) The individuals are arranged with reference to the values of objective function in an ascending manner: $x_{\text {best }}, \ldots, x_{i-1}, x_{i}, x_{i+1}, \ldots, x_{\text {worst }}$

(ii) For each individual $x_{i}$, select two nearest best $\left(x_{i-1}\right)$ and worst $\left(x_{i+1}\right)$ individuals to gain knowledge, and then select another individual randomly $\left(x_{r}\right)$ to share their knowledge. Therefore, the individuals are updated in the following manner, Algorithm 1 (Figure 3).

Step 4 (senior GSK stage): this stage concerns impact and effect of others on an individual. Thus, each individual can be updated by arranging the individual related to the value of the objective function in an ascending order. They are classified into three types of categories as best, middle, or worst individuals:

Best individuals: $100 p \%\left(x_{\mathrm{pb}}\right)$ 


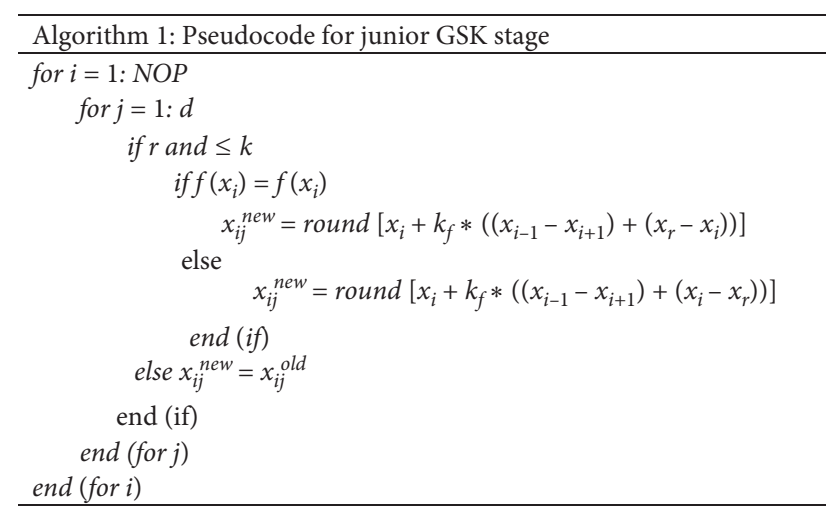

FIgUre 3: Pseudocode for the junior GSK stage.

Middle individual: $d-2 \times 100 p \%\left(x_{m}\right)$

Worst individuals: $100 p \%\left(x_{\mathrm{pw}}\right)$

Where $p \in[0,1]$ is the percentage of best and worst classes.

After classification, select two random vectors from top and bottom $100 p \%$ individuals to gain the knowledge part, and to share the knowledge choose the third vector from the middle individual $d-2 \times 100 p \%$.

The individuals are updated through the pseudocode (Figure 4) and the flowchart of IGSK is presented in Algorithm 2 (Figure 5).

5.2. Constraint Handling Approach. To solve the constrained optimization problem, several techniques have been applied to metaheuristic algorithms [47-49]. Of these, maintaining feasibility of solutions and penalty approaches are most common and popular approaches. However, there are some advantages and disadvantages of these constraint handling techniques. The most common approach with metaheuristic algorithms is the penalty approach [50]. In the penalty approach, a constrained optimization problem is converted into an unconstrained optimization problem by imposing penalty to the infeasible solutions [51]. Usually, the penalty function is not differentiable, and it is the main drawback of this technique [52]. Moreover, they are also required for the suitable penalty factors that estimate the degree of penalization to be applied. Several approaches have been introduced to find the suitable penalty factors such as death penalty, static penalty, adaptive penalty, and dynamic penalty [53]. The other approach is the Lagrangian multiplier method which is based on the Kuhn-Tucker conditions. Several studies have been used: augmented Lagrangian method (ALM) with metaheuristic algorithms. Bahreininejad [54] introduced ALM for the water cycle algorithm and solved the real-time problems. In solving real-world problems, ALM is used with metaheuristic algorithms. To solve structural optimization problem, Adeli and Cheng [55] presented a hybrid genetic algorithm with ALM. In order to optimize the steel structures, Sarma and Adeli [56] used fuzzy ALM with genetic algorithms. Long et al. [57] used ALM with differential evolution algorithm to handle the constraints of engineering problems. Mallipeddi and

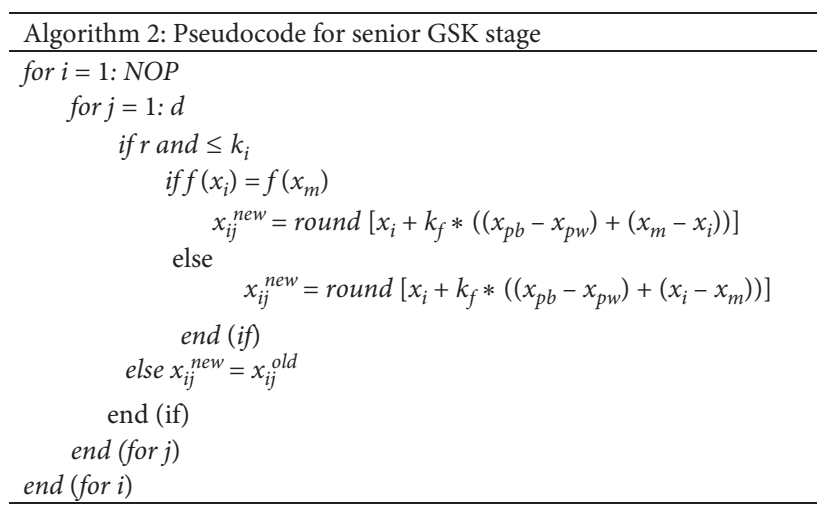

FIgURE 4: Pseudocode for senior GSK stage.

Suganthan [58] used four different constraint handling techniques to solve constrained optimization problems.

In this study, ALM is used, which is like the penalty approach. In ALM, an unconstrained optimization formulation replaces a constrained optimization formulation by the addition of penalty to the former objective function with the Lagrange multiplier parameter. Suppose the constrained optimization problem is given as follows:

$$
\operatorname{Min}=f(X), \quad \text { where }(X)=\left(x_{1}, x_{2}, \ldots, x_{d}\right),
$$

subjected to

$$
g_{i}(X) \leq 0, \quad i=1,2, \ldots, m
$$

When applying the ALM method to the problem, it is changed to an unconstrained optimization one as follows:

$$
\operatorname{Min}=f(X)+\mu \sum_{i=1}^{m}\left(g_{i}(X)\right)^{2}-\lambda \sum_{i=1}^{m}\left(g_{i}(X)\right) \text {, }
$$

where $\mu$ is the penalty parameter, $\sum_{i=1}^{m}\left(g_{i}(X)\right)^{2}$ is quadratic penalty, and $\lambda$ is Lagrange multiplier. In the equation, $\mu$ and $\lambda$ are chosen in such a way that $\lambda$ can remain small to maintain the strategic distance from ill condition. The advantage of ALM is that it decreases the illness ability conditions occurred in the penalty approach.

\section{Computational Results}

The proposed problem has been solved by IGSK, and for comparative evaluation, the problem is also solved by the most popular and well-known algorithms viz. Differential Evolution (DE) [17] and Particle Swarm Optimization (PSO) [19]. The algorithms run over a personal computer Inter ${ }^{\circledR}$ CoreTM i5-7200U CPU @ 2.50 GHz and 4GB RAM. The algorithms are coded on MATLAB R2015a and tested over 30 independent runs. The parameters values of IGSK and the comparative algorithms are presented in Table 3, and results are noted throughout the process. The obtained results are presented in Table 4 in terms of minimum, average, maximum, and median objective function values with standard deviation. The convergence graph of the objective value in relation to the iteration number is shown in Figure 6. 


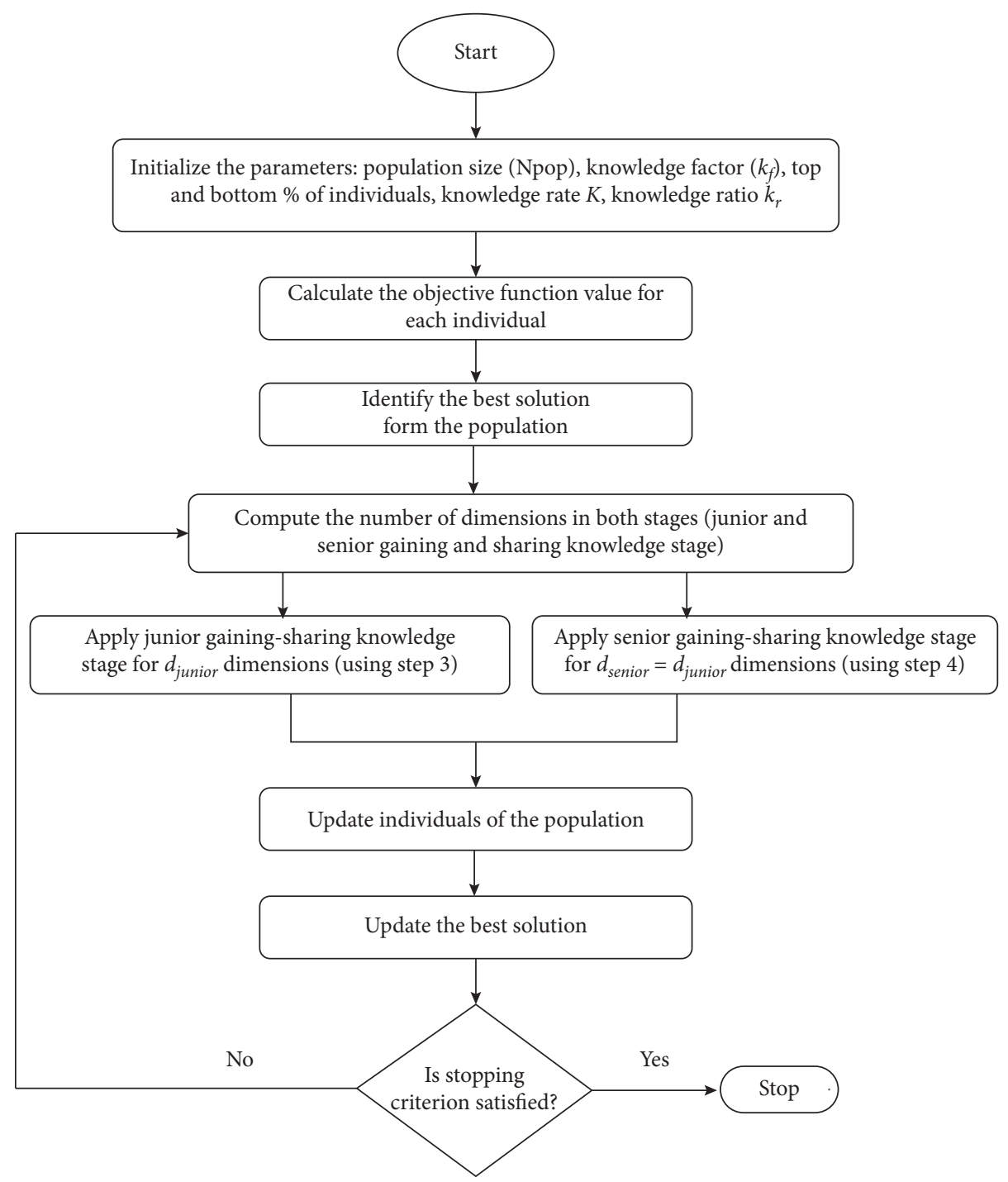

FIGURE 5: The flowchart of IGSK.

TABLE 3: Numerical values of parameters.

\begin{tabular}{lc}
\hline Parameters & Considered values \\
\hline NOP & 100 \\
$k_{f}$ & 0.5 \\
$k_{r}$ & 0.9 \\
$K$ & 10 \\
$P$ & 0.1 \\
Maximum iterations & 100 \\
$\mu$ & $10^{2}$ \\
$\lambda$ & $-10^{4}$ \\
Lower bound of scaling factor for DE & 0.1 \\
Upper bound of scaling factor for DE & 0.9 \\
Crossover probability for DE & 0.75 \\
Constants $c_{1}$ for PSO & 1.5 \\
Constant $c_{2}$ for PSO & 1.5 \\
\hline
\end{tabular}

It is observed in Table 4 that solutions using IGSK are optimal and feasible as constraints are satisfied. The median, mean, and worst objective values $=$ obtained best value, which describes high robustness of IGSK. Besides, Figure 5 displays the convergence of IGSK with other optimizers as the result reaches the optimal value in less count of iterations. It can be deduced that IGSK is an efficient algorithm in terms of rapid convergence, ability in detecting optimal solution, and robustness.

The optimal number of substations and the accumulated ones to be established are shown in Table 5. The total cost for the optimal solution is SR. Table 5 also shows the demanded and the accumulated demanded number of substations.

Figure 7 represents the accumulated number of substations to be built according to the obtained optimal solution, and the accumulated number of demanded substations. As seen in the figure, there is a difference between the two graphs, the total cost given by following the optimum solution is 2,083,425,000 Saudi Riyals (SR), and the total cost for the used protocol of constructing the same number of needed substations in each year is SR $2,104,355,000$. The total cost savings is more than SR 20 million for one city only (Jeddah) which may count for hundreds of millions SR for the whole country. 
TABLE 4: Statistical analysis of obtained solutions of the problem.

\begin{tabular}{lccccc}
\hline Algorithm & Minimum (best) & Median & Mean & Maximum (worst) & St. deviation \\
\hline IGSK & $\mathbf{2 0 8 3 . 4 2 5}$ & 2083.425 & $\mathbf{2 0 8 3 . 4 2 5}$ & 2083.425 & $\mathbf{0}$ \\
DE & 2083.425 & 2085.26 & 2085.342 & 2087.365 & 1.086513 \\
PSO & 2083.68 & 2083.955 & 2083.342 & 2083.955 & 0.173704 \\
\hline
\end{tabular}
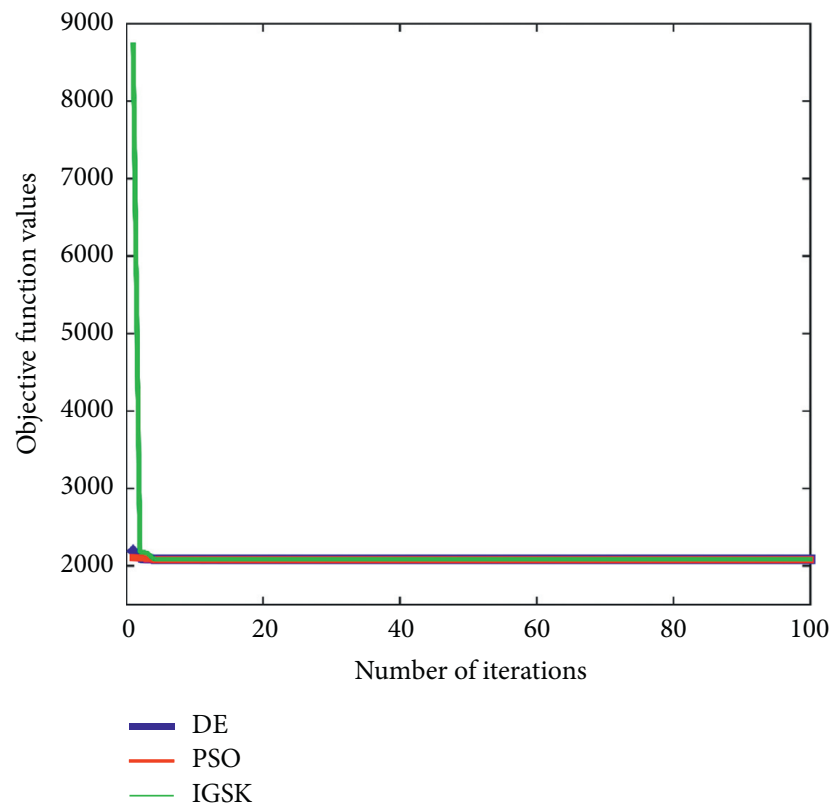

FIgURE 6: The convergence graph of the problem.

TABle 5: Demands and optimum solution for the case study.

\begin{tabular}{lcccccccccc}
\hline Year $y$ & $\mathbf{1}$ & $\mathbf{2}$ & $\mathbf{3}$ & $\mathbf{4}$ & $\mathbf{5}$ & $\mathbf{6}$ & $\mathbf{7}$ & $\mathbf{8}$ & $\mathbf{9}$ & $\mathbf{1 0}$ \\
\hline Optimum solution $\mathbf{x}_{y}$ & 3 & 1 & 3 & 3 & 3 & 3 & 0 & 3 & 3 & 3 \\
Accumulated established substations & 3 & 4 & 7 & 10 & 13 & 16 & 16 & 19 & 22 & 25 \\
Yearly demand $\mathbf{d}_{y}$ & 3 & 1 & 3 & 3 & 2 & 2 & 2 & 3 & 3 & 3 \\
Accumulated demand $\mathbf{D}_{y}$ & 3 & 4 & 7 & 10 & 12 & 14 & 16 & 19 & 22 & 25 \\
\hline
\end{tabular}

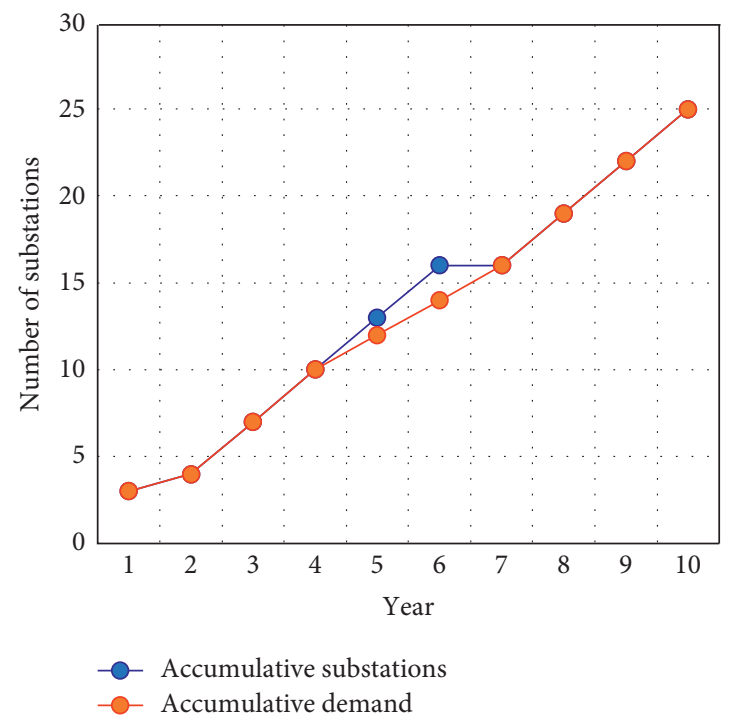

Figure 7: Accumulative number of demanded and established substations. 
For the used case study, the ultimate objective for the problem is to determine the values of the decision variables such that the total costs of building and operating the electric transmission/distribution substations over the long planning horizon are minimized. The decision variables represent the number of substations to be built each year $y$ in the long-term planning horizon, $y=1,2, \ldots, N$. They are denoted as $x_{y}, y=1,2, \ldots, N\left(x_{1}, x_{2}, x_{3}, \ldots, x_{N}\right)$, so we have

$$
\text { Minimize } z=\sum_{y=1}^{N}\left[B_{y}(x) \cdot x_{y}+\left(x_{y} \cdot \sum_{j=y+1}^{N} O_{j}\right)\right] \text {, }
$$

where $B_{y}(x)$ is a function of $x_{\mathbf{y}}, B_{y}^{n}=$ building cost of one substation in year $y$ when buying $n$ substations in that year, this value does not mean the product, where $B_{y}^{n}$ is generally a nonlinear function producing the unit building cost as a function of the number of established substations $x_{y}$ in year $y, y=1,2, \ldots, N, N=$ number of planning years. $O_{y}=$ annual operations and maintenance (O\&M) costs for one substation in year $y$.

Given the values of the parameters $B_{y}(x)$ and $O_{y}$, the total building and operating costs over the long planning horizon reaches the order of billions of Saudi Riyals. The total costs vary in the order of millions of Saudi Rials depending on the number of electric substations to be built each year of the planning horizon. As seen in the obtained solution, there is a difference between the costs associated with the optimum solution and that associated with the used protocol. The total cost given by following the optimum solution is 2,083,425,000 Saudi Riyals (SR) and the total cost for the used protocol of constructing the same number of needed substations in each year is SR 2,104,355,000. The total cost savings is more than SR 20 million for one city only (Jeddah), which may count for hundreds of millions SR for the whole country, which reveals that the proposed algorithm does well on the specific problem at hand.

\section{Conclusions and Points for Future Research Studies}

(1) The total costs of construction and operation of electric stations in a 10-year long-term planning period is optimized by determining the optimal yearly number of stations to be constructed. The decision variables represent the yearly number of stations to be installed. The constraints are the yearly demand and resource constraints.

(2) The problem of formulating the objective function for the various discounted electrical substation prices is to interpolate a polynomial function for the table of points representing the prices and the number of yearly stations in the planning horizon.

(3) The problem is solved using a proposed integer gaining sharing knowledge- (IGSK-) based optimization algorithm. The obtained results clearly show that IGSK gives the optimal solution consistently over all runs. The convergence graph of IGSK establishes that IGSK has rapid convergence to reach the optimal solution and takes very less $(2.03 \mathrm{sec})$ computational time. The optimal cost of constructing the substation in each year is 2,083,425,000 Saudi Riyals (SR) and the number of the substation is $(3,1,3,3,3,3,0,3,3,3)$.

(4) The total cost for the used protocol of constructing the same number of needed substations in each year is SR2, 104, 355, 000. The total cost savings is more than SR20 million.

As future research, the following points are suggested:

(1) Applying the model to other cities and regions of the country and building a comprehensive model for the whole Saudi Arabia and other countries

(2) Performing a parametric analysis to evaluate possible variations in problem factors on the obtained solution

(3) To investigate the changes in money value over the scheduling time period

(4) Building a DSS to assist managers in finding optimum solutions to similar issues

(5) This study can be considered as a basis for planning and scheduling other infrastructure utilities such as power, telecommunication, water, and sewage based on an analytic procedure

(6) To develop alternative adaptive gaining-sharing knowledge-based algorithms

(7) To apply the present approach to the solution of other real-world knowledge-based problems

\section{Data Availability}

No data were used to support this study.

\section{Conflicts of Interest}

The authors declare that the have no conflicts of interest to declare.

\section{Authors' Contributions}

Said Ali Hassan, Khalid Alnowibet, Prachi Agrawal, and Ali Mohamed equally contributed to the study.

\section{Acknowledgments}

This research was funded by Research Supporting Program at King Saud University, Riyadh, Saudi Arabia.

\section{References}

[1] United Nations Website, World Population Projected to Reach 9.7 Billion by 2050, UN, Department of Economic and Social Affairs (DESA), London, UK, 2020.

[2] R. Avtar, S. Tripathi, A. K. Aggarwal, and P. Kumar, "Population-urbanization-energy nexus: a review," Resources, vol. 8, no. 3, p. 136, 2019.

[3] E. Koks, R. Pant, S. Thacker, and J. W. Hall, "Understanding business disruption and economic losses due to electricity 
failures and flooding," International Journal of Disaster Risk Science, vol. 10, pp. 421-438, 2019.

[4] A. W. Mohamed, A. A. Hadi, and A. K. Mohamed, "Gainingsharing knowledge based algorithm for solving optimization problems: a novel nature-inspired algorithm," International Journal of Machine Learning and Cybernetics, vol. 11, pp. 1501-1529, 2020.

[5] Y. Al-Saleh and H. Taleb, "Renewable energy options in Saudi Arabia: the economic viability of solar photovoltaics within the residential sector," in Proceedings of the WSEAS International Conference, Cambridge, UK, 2009.

[6] E. A. Byers, G. Coxon, J. Freer, and J. W. Hall, "Drought and climate change impacts on cooling water shortages and electricity prices in Great Britain," Nature Communications, vol. 11, p. 2239, 2020.

[7] C. W. Gelling, W. L. Barron, J. H. Chamberlain, A. Faruqui, and B. A. Smith, Demand Forecasting for Electric Utilities, Fairmont Press Inc., Libborn, USA, 1992.

[8] A. G. Parols, O. Esmaeil, J. Muthusami, A. Patton, and A. F. Atiya, "Development of an intelligent long-term electric load forecasting system", intelligent systems applications to power systems," in Proceedings of the ISAP International Conference, pp. 288-292, Cambridge, UK, 2009.

[9] F. C. Lu and Y. Y. Hsu, "Reactive power/voltage control in a distribution substation using dynamic programming," IEE Proceedings, Generation, Transmission and Distribution, vol. 142, no. 6, pp. 639-645, 1995.

[10] Y. Yixin, C. Wang, G. Shaoyun, J. Xiao, X. Yan, and C. Huang, Models and Methods for Urban Distribution Planning, School of Electrical and Automation Engineering, Tianjin University, Perth, WA, Australia, 2000.

[11] K. Nagasaka and M. Al-Mamun, "Long-term peak demand prediction of 9 Japanese power utilities using radial basis function networks," Power Engineering Society General Meeting, IEEE, vol. 1, pp. 315-322, 2004.

[12] S. S. Al-Ghamdi, A Dynamic Programming Model with Parametric Study for Scheduling the Construction of Electric Transmission/Distribution Substations in Jeddah City, Department of Industrial Engineering, Faculty of Engineering, King Abdulaziz University, Jeddah, 2010.

[13] S. A. Hassan, I. A. El-Darrab, and M. A. Al-Ameer, "A Dynamic Programming Model for Scheduling the Electric Transmission/Distribution Substations in Jeddah City," in Proceedings of the WSEAS International Conference, Cambridge, UK, 2009.

[14] S. A. El Quliti, I. A. El-Darrab, and M. A. Al-Ameer, A Dynamic Programming Model for Scheduling the Electric Transmission/Distribution Substations in Jeddah City, University of Cambridge, Cambridge, UK, 2009.

[15] S. A. El-Quliti, and M. R. Kabli, "Economic scheduling of the electric transmission/distribution substations in Jeddah city with parametric results," Journal of King Abdulaziz University, Engineering Sciences, vol. 23, no. 2, pp. 115-136, 2012.

[16] J. Holland, Adaptation in Natural and Artificial Systems: An Introductory Analysis with Application to Biology. Control and Artificial Intelligence, University of Michigan Press, Michigan, USA, 1975.

[17] R. Storn, and K. Price, "Differential evolution-a simple and efficient heuristic for global optimization over continuous spaces," Journal of Global Optimization, vol. 11, no. 4, pp. 341-359, 1997.

[18] O. B. Haddad, M. Moravej, and H. A. Loáiciga, “Application of the water cycle algorithm to the optimal operation of reservoir systems," Journal of Irrigation and Drainage Engineering, vol. 141, no. 5, Article ID 04014064, 2015.

[19] R. Eberhart and J. Kennedy, "A new optimizer using particle swarm theory," in Proceedings of the Sixth International Symposium on Micro Machine and Human Science, pp. 39-43, Cambridge, UK, 2009.

[20] M. Dorigo, V. Maniezzo, and A. Colorni, "Ant system: optimization by a colony of cooperating agents," IEEE Transactions on Systems, Man, and Cybernetics-Part B: Cybernetics, vol. 26, no. 1, pp. 29-41, 1996.

[21] S. Mirjalili and A. Lewis, "The whale optimization algorithm," Advances in Engineering Software, vol. 95, pp. 51-67, 2016.

[22] S. Kirkpatrick, C. D. Gelatt, and M. P. Vecchi, "Optimization by simulated annealing," Science, vol. 220, no. 4598, pp. 671-680, 1983.

[23] Z. W. Geem, J. H. Kim, and G. V. Loganathan, "A new heuristic optimization algorithm: harmony search," Simulation, vol. 76, no. 2, pp. 60-68, 2001.

[24] H. Eskandar, A. Sadollah, A. Bahreininejad, and M. Hamdi, "Water cycle algorithm-a novel metaheuristic optimization method for solving constrained engineering optimization problems," Computers \& Structures, vol. 110-111, pp. 151-166, 2012.

[25] L. M. Zhang, C. Dahlmann, and Y. Zhang, "Human-inspired algorithms for continuous function optimization," in Proceedings of the 2009 IEEE International Conference on Intelligent Computing and Intelligent Systems, pp. 318-321, Cambridge, UK, 2009.

[26] R. V. Rao, V. J. Savsani, and D. P. Vakharia, "Teaching-learning based optimization: an optimization method for continuous nonlinear large scale problems," Information Sciences, vol. 183, no. 1, pp. 1-15, 2012.

[27] L. Giraud-Moreau and P. Lafon, "A comparison of evolutionary algorithms for mechanical design components," Engineering Optimization, vol. 34, no. 3, pp. 307-322, 2002.

[28] H. Youssef, S. M. Sait, and H. Adiche, "Evolutionary algorithms, simulated annealing and tabu search: a comparative study," Engineering Applications of Artificial Intelligence, vol. 14, no. 2, pp. 167-181, 2001.

[29] S. A. El-Qulity and A. W. Mohamed, "A generalized national planning approach for admission capacity in higher education: a nonlinear integer goal programming model with a novel differential evolution algorithm," Computational Intelligence and Neuroscience, vol. 2016, p. 14, Article ID 5207362, 2016.

[30] S. A. El-Quliti, A. H. Ragab, R. Abdelaal et al., "A nonlinear goal programming model for university admission capacity planning with modified differential evolution algorithm," Mathematical Problems in Engineering, vol. 2015, p. 13, Article ID 892937, 2015.

[31] S. A. El-Quliti, and A. W. Mohamed, "A large-scale nonlinear mixed-binary goal programming model to assess candidate locations for solar energy stations: an improved binary differential evolution algorithm with a case study," Journal of Computational and Theoretical Nanoscience, vol. 13, no. 11, pp. 7909-7921, 2016.

[32] A. W. Mohamed, H. Z. Sabry, and M. Khorshid, "An alternative differential evolution algorithm for global optimization," Journal of Advanced Research, vol. 3, no. 2, pp. 149-165, 2012.

[33] A. W. Mohamed, "Solving stochastic programming problems using new approach to differential evolution algorithm," Egyptian Information Journal, vol. 18, no. 2, pp. 75-86, 2017. 
[34] A. W. Mohamed, A. K. Mohamed, E. Z. Elfeky, and M. Saleh, "Enhanced directed differential evolution algorithm for solving constrained engineering optimization problems," International Journal of Applied Metaheuristic Computing (IJAMC), vol. 10, no. 1, pp. 1-28, 2019.

[35] A. W. Mohamed, "A new modified binary differential evolution algorithm and its applications," Applied Mathematics \& Information Sciences, vol. 10, no. 5, pp. 1965-1969, 2016.

[36] A. Wagdy Mohamed, H. Z. Sabry, and A. Farhat, "Advanced differential evolution algorithm for global numerical optimization," in Proceedings of the IEEE International Conference on Computer Applications and Industrial Electronics (ICCAIE '11), pp. 156-161, Penang, Malaysia, December 2011.

[37] A. K. Mohamed, A. W. Mohamed, E. Z. Elfeky, and M. Saleh, "Solving constrained non-linear integer and mixed-integer global optimization problems using enhanced directed differential evolution algorithm," in Machine Learning Paradigms: Theory and ApplicationSpringer, Cham, Switzerland, 2019.

[38] A. H. M. Ragab, S. A. El-Quliti, R. Abdelaal et al., "Higher education admission capacity planning using a large scale nonlinear integer goal programming model with improved differential evolution algorithm," Journal of Computational and Theoretical Nanoscience, vol. 13, no. 11, pp. 7864-7878, 2016.

[39] A. W. Mohamed, "An efficient modified differential evolution algorithm for solving constrained non-linear integer and mixed-integer global optimization problems," International Journal of Machine Learning and Cybernetics, vol. 8, no. 3, pp. 989-1007, 2017.

[40] L. Cheng, Y. Wang, C. Wang, A. W. Mohamed, and T. Xiao, "Adaptive differential evolution based on successful experience information," IEEE Access, vol. 8, pp. 164611-164636, 2020.

[41] E. Chen, J. Chen, A. W. Mohamed, B. Wang, Z. Wang, and Y. Chen, "Swarm intelligence application to UAV aided IoT data acquisition deployment optimization," IEEE Access, vol. 8, pp. 175660-175668, 2020.

[42] H. M. Gomes, "Truss optimization with dynamic constraints using a particle swarm algorithm," Expert Systems with Applications, vol. 38, no. 1, pp. 957-968, 2011.

[43] S. Wang and J. Watada, "Capacitated two-stage facility location problem with fuzzy costs and demands," International Journal of Machine Learning and Cybernetics, vol. 4, no. 1, pp. 65-74, 2013.

[44] D. P. Kanoussis, "Polynomial Equations: Systematic Theory Summary, Challenging Examples and Problems," 2019.

[45] T. Vialar, Handbook of Mathematics, Library of Congress, London, UK, 2017.

[46] MyAssays Ltd, My Curve Fit Software, on Line Curve Fitting, 2017.

[47] K. Deb, "An efficient constraint handling method for genetic algorithms," Computer Methods in Applied Mechanics and Engineering, vol. 186, no. 2-4, pp. 311-338, 2000.

[48] C. A. C. Coello, "Theoretical and numerical constrainthandling techniques used with evolutionary algorithms: a survey of the state of the art," Computer Methods in Applied Mechanics and Engineering, vol. 191, no. 11-12, pp. 1245-1287, 2002.

[49] N. Muangkote, L. Photong, and A. Sukprasert, "Effectiveness of constrained handling techniques of improved constrained differential evolution algorithm applied to constrained optimization problems in mechanical engineering," ITMSOC
Transactions on Innovation \& Business Engineering, vol. 4, pp. 1-21, 2019.

[50] R. Courant, "Variational methods for the solution of problems of equilibrium and vibrations," Lecture Notes in Pure and Applied Mathematics, vol. 4, 1994.

[51] S. G. Nash, Linear and Nonlinear Programming, McGraw-Hill Science, Engineering \& Mathematics, London, UK, 1996.

[52] A. E. Smith, and D. W. Coit, "Penalty functions," Handbook of Evolutionary Computation, vol. 97, no. 1, 1997.

[53] E. Mezura-Montes and C. A. C. Coello, "An empirical study about the usefulness of evolution strategies to solve constrained optimization problems," International Journal of General Systems, vol. 37, no. 4, pp. 443-473, 2008.

[54] A. Bahreininejad, "Improving the performance of water cycle algorithm using augmented Lagrangian method," Advances in Engineering Software, vol. 132, pp. 55-64, 2019.

[55] H. Adeli and N. T. Cheng, "Augmented Lagrangian genetic algorithm for structural optimization," Journal of Aerospace Engineering, vol. 7, no. 1, pp. 104-118, 1994.

[56] K. C. Sarma and H. Adeli, "Fuzzy genetic algorithm for optimization of steel structures," Journal of Structural Engineering, vol. 126, no. 5, pp. 596-604, 2000.

[57] W. Longa, X. Liang, Y. Huang, and Y. Chen, "A hybrid differential evolution augmented Lagrangian method for constrained numerical and engineering optimization," Computer-Aided Design, vol. 45, pp. 1562-1574, 2013.

[58] R. Mallipeddi and P. N. Suganthan, "Ensemble of constraint handling techniques," IEEE Transactions on Evolutionary Computation, vol. 14, no. 4, pp. 561-579, 2010. 